\title{
Diagnostic value of cyclin-dependent kinase/cyclin-dependent kinase inhibitor expression ratios as biomarkers of locoregional and hematogenous dissemination risks in oral squamous cell carcinoma
}

\author{
MASAKI NAGATA $^{1}$, HIROSHI KURITA ${ }^{2}$, KOHYA UEMATSU $^{1}$, SHIN OGAWA ${ }^{1}$, \\ KATSU TAKAHASHI ${ }^{3}$, HIDEYUKI HOSHINA ${ }^{1}$ and RITSUO TAKAGI ${ }^{1}$
}

\begin{abstract}
${ }^{1}$ Division of Oral and Maxillofacial Surgery, Niigata University Graduate School of Medical and Dental Sciences, Niigata, Niigata $951-8514 ;{ }^{2}$ Department of Dentistry and Oral Surgery, Shinshu University School of Medicine, Matsumoto, Nagano 390-8621; ${ }^{3}$ Department of Oral and Maxillofacial Surgery, Kyoto University,
\end{abstract} Graduate School of Medicine, Kyoto, Kyoto 606-8507, Japan

Received February 13, 2015; Accepted March 12, 2015

DOI: $10.3892 / \mathrm{mco} .2015 .578$

\begin{abstract}
The aim of the present study was to investigate the diagnostic value of cell cycle-related genes in oral squamous cell carcinoma (OSCC) by examining the expression of the following genes in 77 OSCC tissues by quantitative polymerase chain reaction: Cyclin genes (CCNA1, CCNDI, $C C N D 2$ and $C C N E 1$ ), cyclin-dependent kinase (CDK) genes (CDK1, CDK2 and CDK4), CDK inhibitor genes (CDKN2A, $C D K N 1 A, C D K N 1 B$ and $C D K N 1 C$ ), and integrin and associated genes that we previously reported (ITGA3, ITGB4, CD9 and $J U P)$. The expression ratios of 66 combinations of the 11 cell cycle-related genes were analyzed to examine their associations with major clinical events using Mann-Whitney $\mathrm{U}$ and log-rank tests. Three expression ratios $(C D K 1 / C D K N 1 B$, $C D K 2 / C D K N 1 A$ and $C C N E 1 / C D K 2)$ showed associations on univariate analyses and their diagnostic value was re-analyzed with integrin gene expression biomarkers (ITGA3/CD 9 and ITGB4/JUP) using the Cox proportional hazards model and Kaplan-Meier estimates. Lymph node metastasis occurred in $>90 \%$ of double-positive cases
\end{abstract}

Correspondence to: Dr Masaki Nagata, Division of Oral and Maxillofacial Surgery, Niigata University Graduate School of Medical and Dental Sciences, Gakkocho-dori 2-5274, Niigata, Niigata 951-8514, Japan

E-mail: nagatam@dent.niigata-u.ac.jp

Abbreviations: OSCC, oral squamous cell carcinoma; LNM, lymph node metastasis; PSR, primary site recurrence; DSD, disease-specific death; K-M curve, Kaplan-Meier curve; CDK, cyclin-dependent kinase; CDKI, cyclin-dependent kinase inhibitor; RT-qPCR, reverse transcription-quantitative polymerase chain reaction

Key words: squamous cell carcinoma, biomarker, cyclin-dependent kinase inhibitor, cyclin-dependent kinase, lymph node metastasis, primary site recurrence, distant metastasis, integrin $\alpha 3$, integrin $\beta 4$
(high-ITGA3/CD9 and high-CDK1/CDKN1B) irrespective of tumor size $(\mathrm{P}<0.0001)$. Primary site recurrence was found in $>30 \%$ of double-positive cases (high-ITGA3/CD 9 and high-CDK2/CDKN1A) with tumors $>20 \mathrm{~mm}(\mathrm{P}=0.003)$. Triple-positive (high-ITGB4/JUP, high-ITGA3/CD9 and high-CDK2/CDKN1A) was associated with distant metastasis $(\mathrm{P}<0.0001)$, but not with other clinical parameters. Disease-specific death occurred in $55 \%$ of double-positive cases (high-ITGA3/CD 9 and high-CDK2/CDKN1A) $(\mathrm{P}<0.0001)$ and a positive surgical margin was a significant factor for fatality in these cases. Reliable prediction of locoregional and hematogenous dissemination risks in OSCC using the four CDK and integrin gene expression ratios is a promising biomarker system. Clinical use of these parameters may improve the control rate with the use of new therapeutic strategies.

\section{Introduction}

Over $90 \%$ of lip and oral cavity cancers are squamous cell carcinomas, with 300,000 new cases annually worldwide. Based on the classification of the cancer cell type, it is associated with squamous cell carcinoma of the head and neck, esophagus, lung, cervix, uterus, skin and mucosa at other sites; almost 2 million new cases of these types of cancer are reported each year (1). Locoregional treatment is frequently used for squamous cell carcinoma and the standard treatment currently consists of surgery plus adjuvant chemoradiation. Despite the recent introduction of molecular-targeted therapy, treatment options depend on the disease stage (2). Direct cause of fatality from oral squamous cell carcinoma (OSCC) is uncontrollable locoregional recurrence at the surgically treated site (such as resection of primary cancer and neck dissection) or distant metastasis. Locoregional and hematogenous dissemination in the early stage of cancer also remains difficult to control with standard treatment (3). To improve the control rate, local plus systemic treatment strategies against micro-metastasis are required from the outset of treatment. 
Our previous studies conducted comprehensive integrin gene expression analysis to identify prognostic biomarkers that predict the clinical behavior of OSCC and identified that the two gene expression ratios, ITGA3/CD9 and ITGB4/JUP, reflect malignant behavior associated with lymph node metastasis (LNM) and distant metastasis, respectively (3-5). In the present study, the diagnostic value of cyclins, cyclin-dependent kinases (CDKs) and CDK inhibitors (CDKIs) gene expression was investigated since these molecules play central roles in cell cycle control, have been extensively studied, mediate carcinogenesis and can be used as prognostic factors of cancer $(6,7)$. These genes became of particular interest recently as possible molecular targets of cancer therapy (8). Therefore, the association of the expression of 11 cyclin-related genes in OSCC tissues with clinical events were investigated to examine their value as biomarkers.

\section{Materials and methods}

Patients and specimens. Tumor samples were collected at biopsy from 77 patients with OSCC (49 men, 28 women; mean age, 66 years; range, 22-86 years) who underwent surgical treatment between 1999 and 2012 at the Dental Department of Niigata University Medical and Dental Hospital (Niigata, Japan); the Special Dental Care and Oral Surgery Department of Shinshu University Hospital (Nagano, Japan); and the Division of Oral Surgery of Nagaoka Red Cross Hospital (Nagaoka, Japan) (Table I). The treatment modalities included local resection, composite resection (resection of primary oral cancer, a portion of the oral floor and mandible, and reconstruction with tissue transplantation and neck dissection) and composite resection with radiation therapy with or without intravenous adjuvant chemotherapy.

The study was performed in accordance with the guidelines of the Declaration of Helsinki and the protocol was approved by the Research Ethics Committee of Niigata University Medical and Dental Hospital, the Research Ethics Committee of Shinshu University Hospital and the Ethics Committee of Nagaoka Red Cross Hospital. Patients provided written informed consent to participate in the study.

Total RNA extraction from carcinoma tissue. Cancer tissue specimens were preserved by immersion in RNAlater solution (Invitrogen Life Technologies, Tokyo, Japan). Extraction of total RNA was performed using the RNeasy Lipid Tissue Mini kit (Qiagen, Tokyo, Japan) following homogenization by TissueLyserLT(Qiagen)inQIAzolLysis reagentaccording tothe manufacturer's instructions. Synthesis of first-strand cDNA was performed by reverse transcription using total RNA $(0.2-1 \mu \mathrm{g})$ as a template (SuperScript III ${ }^{\circledR}$; Invitrogen Life Technologies).

Gene expression analysis by reverse transcription-quantitative polymerase chain reaction $(R T-q P C R)$. RT-qPCR was performed on a Thermal Cycler Dice ${ }^{\circledR}$ Real Time System II (Takara Bio Inc., Shiga, Japan) using cDNA synthesized from the patients' cancer specimens and TaqMan probes for cyclins, CDKs, CDKIs, integrins and integrin-related genes (TaqMan Gene Expression Assays; Invitrogen Life Technologies) according to the following protocol: $600 \mathrm{sec}$ at $95^{\circ} \mathrm{C}$, followed by extension for $15 \mathrm{sec}$ at $95^{\circ} \mathrm{C}$ and $60 \mathrm{sec}$ at $60^{\circ} \mathrm{C}$. Relative
Table I. Demographic and clinicopathological characteristics of 77 patients with oral squamous cell carcinoma.

\begin{tabular}{lc} 
Clinicopathological factors & Values \\
\hline Total, $\mathrm{n}$ & 77
\end{tabular}

Observation period, days [range (mean)]

$144-2,762(1,510)$

Age, years [range (mean)]

$22-86(66)$

Gender, n (\%)

Male

$49(63.6)$

Female

$28(36.4)$

Site, $\mathrm{n}(\%)$

Tongue

38 (49.4)

Gingiva

$27(35.1)$

Oral floor

$9(11.7)$

Buccal mucosa

Tumor size ${ }^{\mathrm{a}}$, mm [range (mean)]

8-52 (27.1)

$\leq 20 \mathrm{~mm}, \mathrm{n}(\%)$

$25(32.5)$

21-30 mm, n (\%)

$26(33.8)$

$18(23.4)$

$8(10.4)$

$>40 \mathrm{~mm}, \mathrm{n}(\%)$

$24(31.2)$

$35(45.5)$

$3(3.9)$

$15(19.5)$

$\mathrm{T} 4$

$37(48.1)$

$10(13.0)$

$30(39.0)$

$0(0.0)$

N3

$31(40.3)$

$46(59.7)$

$72(93.5)$

$5(6.5)$

$71(92.2)$

Positive

Positive
$14(18.2)$
Alive

${ }^{a}$ Major width of the tumor. ${ }^{b}$ Tumor $(\mathrm{T})$; and ${ }^{\mathrm{c} l y m p h}$ node $(\mathrm{N})$ category according to the Union for International Cancer Control Tumor-Node-Metastasis classification of malignant tumors of the lip and oral cavity. ${ }^{\mathrm{d}}$ Histopathological classification of oral squamous cell carcinoma (YK grade) according to Yamamoto et al (9). ${ }^{\mathrm{e}} \mathrm{Histological}$ tumor positiveness of the surgical margin. ${ }^{\mathrm{f} F a t a l i t y}$ outcome from uncontrollable oral squamous cell carcinoma. 
Table II. Final log-rank test results for the risks of lymph node metastasis, primary site recurrence, distant metastasis and disease-specific death in oral squamous cell carcinoma.

\begin{tabular}{|c|c|c|c|c|}
\hline Log-rank (Mantel-Cox) & $\begin{array}{l}\text { Lymph node } \\
\text { metastasis }\end{array}$ & $\begin{array}{l}\text { Primary site } \\
\text { recurrence }\end{array}$ & $\begin{array}{c}\text { Distant } \\
\text { metastasis }\end{array}$ & $\begin{array}{c}\text { Disease-specific } \\
\text { death }^{\mathrm{d}}\end{array}$ \\
\hline \multicolumn{5}{|l|}{ Gene expression ratios } \\
\hline$C D K 2 / C D K N 1 A$ & - & 0.019 & 0.017 & 0.002 \\
\hline$C D K 1 / C D K N 1 B$ & 0.008 & 0.047 & - & 0.041 \\
\hline$C C N E 1 / C D K 2$ & 0.002 & - & 0.012 & 0.005 \\
\hline \multicolumn{5}{|c|}{ Integrin gene expression ratios } \\
\hline ITGB4/JUP & - & - & 0.008 & - \\
\hline$I T G A 3 / C D 9$ & 0.005 & - & - & 0.011 \\
\hline \multicolumn{5}{|c|}{ Clinicopathological parameters } \\
\hline Size, $>20 \mathrm{~mm}^{\mathrm{a}}$ & 0.047 & 0.057 & - & - \\
\hline Positive margin ${ }^{\mathrm{b}}$ & 0.002 & $<0.001$ & - & 0.001 \\
\hline Invasive histolog $y^{c}$ & 0.005 & 0.032 & 0.051 & 0.001 \\
\hline
\end{tabular}

standard curves representing several 10-fold cDNA dilutions (1:10:100:1,000:10,000:100,000) from an OSCC tissue sample were used for the linear regression analysis of other samples. The manufacturer's TaqMan probe assay IDs are as follows: CCNA1: Hs00171105_m1; CCND1: Hs00277039_m1; CCND2: Hs00153380_m1; CCNE1: Hs00233356_m1; CDK1: Hs00938777_m1; CDK2: Hs00608082_m1; CDK4: Hs00364847_m1; CDKN2A: Hs00923894_m1; CDKN1A: Hs00355782_m1; CDKN1B: Hs01597588_m1; CDKN1C: Hs00175938_m1;ITGA3:Hs00233722_m1;ITGB4:Hs01103172_g1; CD9: Hs01124027_m1; JUP: Hs00158408_m1; and GAPDH: Hs02758991_g1.

Statistical analysis. Expression ratios of all the possible combinations of the 12 genes (11 cyclin-related genes and GAPDH; 66 combinations in total) and expression ratios of two combinations of the integrin genes (ITGA3/CD9 and ITGB4/JUP) were calculated for each of the 77 OSCC cases. Each gene expression ratio was categorized by the cut-off point estimated from the receiver operating characteristic curve for each clinical event. The response variables used were LNM determined by histopathological examination of the surgical specimen, primary site recurrence (PSR) following surgery, distant metastasis following successful surgery for the primary cancer and disease-specific death (DSD) from uncontrollable OSCC. The clinicopathological parameters were age, gender, tumor size, tumor category (Union for International Cancer Control Tumor-Node-Metastasis Classification), histopathological mode of invasion (9) and positive surgical margins on histological examination. Distant metastasis following locoregional failure was regarded as locoregional recurrence.

The influence of the gene expression ratios and clinicopathological parameters on LNM, PSR, distant metastasis and DSD was examined by univariate analyses (Mann-Whitney U test followed by log-rank test) to optimize the combination of variables for subsequent multivariate analysis. Analyses using a Cox proportional hazards model and Kaplan-Meier (K-M) curves were performed for the events of LNM, PSR, distant metastasis and DSD (or disease-specific survival in K-M curves) using SPSS 21.0 (IBM Japan Ltd., Tokyo, Japan). Time to each event was calculated from the date of first visit to the date of neck dissection, diagnosis of recurrence and date of DSD or final observation for surviving patients. $\mathrm{P}<0.05$ was considered to indicate a statistically significant difference.

\section{Results}

Univariate analysis. The gene expression ratios and clinicopathological parameters that showed significance for each clinical event on the Mann-Whitney U test were further tested by the log-rank test. This revealed that the four clinical events were influenced by three CDK-related gene expression ratios, as well as the two integrin gene expression ratios, tumor size, surgical margin status and invasive histology (Table II). For multivariate analysis, these gene expression ratios were used in double or triple combinations to group 'double-positive' or 'triple-positive' cases.

LNM. In the Cox proportional hazards model of LNM (Table IIIA), the single parameters (high-ITGA3/CD9 and high- $C D K 1 / C D K N 1 B$ ) were independent significant variables in addition to tumor size (size, $>20 \mathrm{~mm}$ ). The rate of LNM was represented by the K-M curve (one minus cumulative survival) between groups of double-positive cases (high-ITGA3/CD9 and high- $C D K 1 / C D K N 1 B$ ) and the remaining (negative) cases according to size ( $\leq 20$ or $>20 \mathrm{~mm}$ ) (Fig. 1A). The double-positive 
Table III. Cox proportional hazard models for the risks of lymph node metastasis, primary site recurrence, distant metastasis and fatality in oral squamous cell carcinoma.

A, Lymph node metastasis

\begin{tabular}{|c|c|c|c|c|c|c|c|}
\hline \multirow[b]{2}{*}{ Variable } & \multirow[b]{2}{*}{$\mathrm{B}$} & \multirow[b]{2}{*}{ SE } & \multirow[b]{2}{*}{ Wald } & \multirow[b]{2}{*}{ P-value } & \multirow[b]{2}{*}{ OR } & \multicolumn{2}{|c|}{$95 \% \mathrm{CI}$ for $\mathrm{OR}$} \\
\hline & & & & & & Lower limit & Upper limit \\
\hline High-ITGA3/CD9 & 1.057 & 0.341 & 9.627 & 0.002 & 2.878 & 1.476 & 5.613 \\
\hline High- $C D K 1 / C D K N 1 B$ & 0.853 & 0.332 & 6.609 & 0.010 & 2.346 & 1.225 & 4.494 \\
\hline Size $>20 \mathrm{~mm}^{\mathrm{a}}$ & 0.623 & 0.367 & 2.875 & 0.090 & 1.864 & 0.908 & 3.829 \\
\hline
\end{tabular}

B, Primary site recurrence

$95 \%$ CI for OR

\begin{tabular}{lccccccc}
\cline { 6 - 7 } Variable & $\mathrm{B}$ & $\mathrm{SE}$ & Wald & P-value & OR & Lower limit & Upper limit \\
\hline $\begin{array}{l}\text { High-ITGA3/CD9 and } \\
\text { High-CDK2/CDKN1A }\end{array}$ & 2.352 & 0.883 & 7.089 & 0.008 & 10.505 & 1.860 & 59.328 \\
$\begin{array}{l}\text { Positive margin } \\
\text { b }\end{array}$ & 2.291 & 0.903 & 6.444 & 0.011 & 9.886 & 1.686 & 57.980 \\
\hline
\end{tabular}

C, Distant metastasis

$95 \%$ CI for OR

\begin{tabular}{|c|c|c|c|c|c|c|c|}
\hline \multirow[b]{2}{*}{ Variable } & \multirow[b]{2}{*}{ B } & \multirow[b]{2}{*}{ SE } & \multirow[b]{2}{*}{ Wald } & \multirow[b]{2}{*}{ P-value } & \multirow[b]{2}{*}{ OR } & \\
\hline & & & & & & Lower limit & Upper limit \\
\hline $\begin{array}{l}\text { High-ITGB4/JUP, } \\
\text { High-ITGA3/CD } 9 \text { and } \\
\text { High-CDK2/CDKN1A }\end{array}$ & 3.350 & 1.119 & 8.960 & 0.003 & 28.496 & 3.179 & 255.464 \\
\hline
\end{tabular}

D, Disease-specific death ${ }^{c}$

$95 \%$ CI for OR

\begin{tabular}{|c|c|c|c|c|c|c|c|}
\hline \multirow[b]{2}{*}{ Variable } & \multirow[b]{2}{*}{ B } & \multirow[b]{2}{*}{ SE } & \multirow[b]{2}{*}{ Wald } & \multirow[b]{2}{*}{$\mathrm{P}$-value } & \multirow[b]{2}{*}{ OR } & \\
\hline & & & & & & Lower limit & Upper limit \\
\hline $\begin{array}{l}\text { High-ITGA3/CD } 9 \text { and } \\
\text { High-CDK2/CDKN } 1 A\end{array}$ & 2.330 & 0.569 & 16.750 & $<0.001$ & 10.274 & 3.367 & 31.350 \\
\hline Positive margin & 1.919 & 0.686 & 7.835 & 0.005 & 6.817 & 1.778 & 26.139 \\
\hline
\end{tabular}

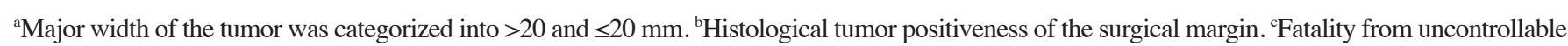
oral squamous cell carcinoma. Cox Proportional Hazard Models for the risks of (A) lymph node metastasis, (B) primary site recurrence, (C) distant metastasis and (D) fatality outcome from oral squamous cell carcinoma. Continuous variables of gene expression ratios are categorized according to the cut-off points introduced by receiver operating characteristic curves of lymph node metastasis for ITGA3/CD9, of distant metastasis for $C D K 2 / C D K N 1 A$ and ITGB4/JUP. B, regression coefficient; SE, standard error; OR, odds ratio; CI, confidence interval; $C D K$, cyclin-dependent kinase.

cases (high-ITGA3/CD9 and high-CDK1/CDKN1B) consistently exhibited a high rate of LNM $(>85 \%)$ irrespective of tumor size, but the negative cases revealed an increasing rate of LNM with larger tumor size (>20 mm).

PSR. The Cox proportional hazards model identified double-positive (high-ITGA3/CD9 and high-CDK2/CDKN1A) and positive margin to be independent significant variables for PSR (Table IIIB). Although positive margin is a clinical event following surgery, it was included in the analysis due to its considerable influence on the risk for PSR. The rate of PSR was represented by the K-M curves (one minus cumulative survival) between groups of double-positive cases (high-ITGA3/CD9 and high-CDK2/CDKN1A) and the remaining negative cases according to size, $\leq 20$ or $>20 \mathrm{~mm}$ (Fig. 1B). The double-positive group (high-ITGA3/CD9 and high- $C D K 2 / C D K N 1 A)>20 \mathrm{~mm}$ in size clearly showed a higher rate of PSR independent of the primary therapeutic 
A

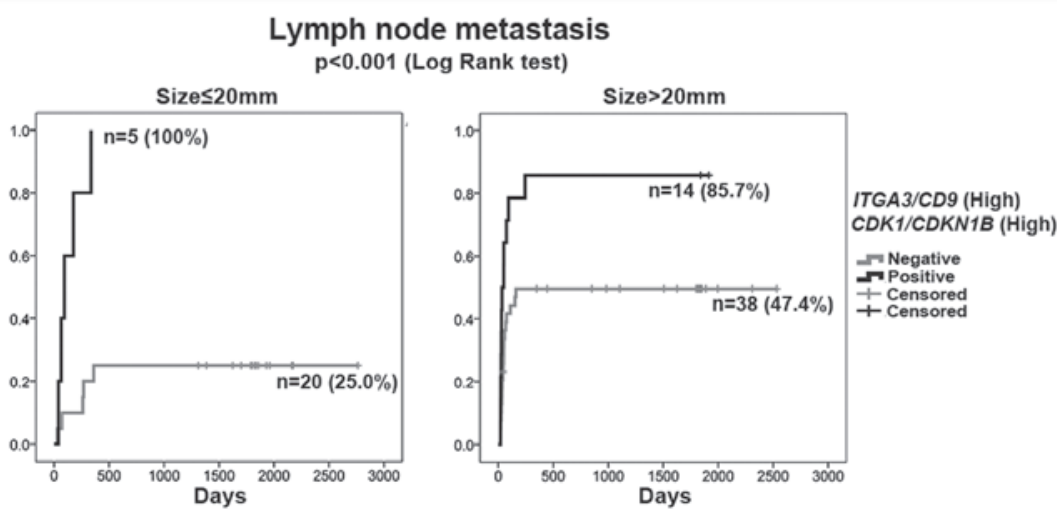

B

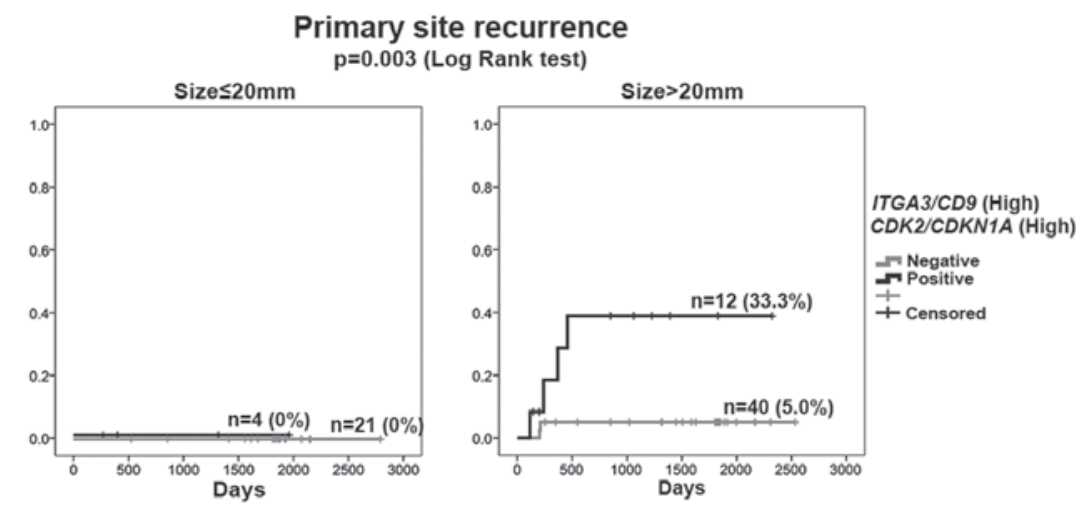

C

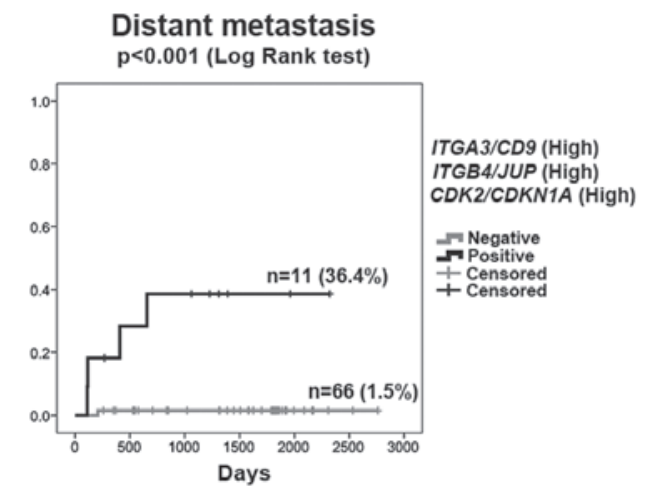

D

Disease-specific survival $\mathrm{p}<0.001$ (Log Rank test)
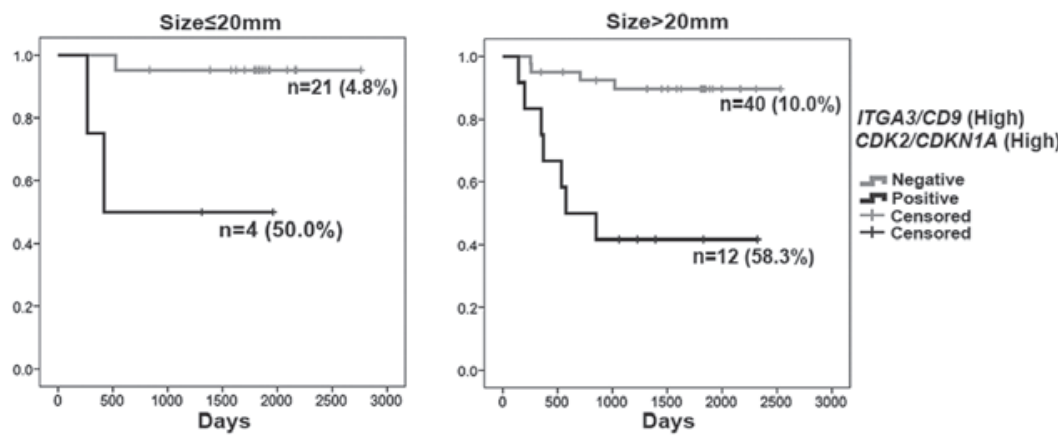

Figure 1. Kaplan-Meier curves for 77 patients with oral squamous cell carcinoma. (A) Lymph node metastasis, (B) primary site recurrence, (C) distant metastasis and (D) disease-specific survival. (A-C) One minus cumulative survival and (D) cumulative survival. Each outcome (A, B and D) was stratified by size $(\leq 20$ or $>20 \mathrm{~mm})$.

intervention (positive margin), indicating diagnostic value of the high-ITGA3/CD 9 and high-CDK2/CDKN1A as a biomarker at the outset of treatment planning.
Distant metastasis. The Cox proportional hazards model identified that triple-positive (high-ITGB4/JUP, high-ITGA3/CD9 and high-CDK2/CDKN1A) was the only 
significant variable for distant metastasis (Table IIIC). Tumor size, histopathological parameters and LNM did not exhibit a significant influence in multivariate analysis in the presence of the triple-positive (high-ITGB4/JUP, high-ITGA3/CD9 and high-CDK2/CDKN1A) influence. The rate of distant metastasis was presented by the K-M curves (one minus cumulative survival) between the groups of triple-positive cases (high-ITGB4/JUP, high-ITGA3/CD9 and high-CDK2/CDKN1A) and the remaining negative cases (Fig. 1C). Among all 77 cases, 4 of 5 cases that developed distant metastasis were extracted from 11 cases based on their triple-positive status (high-ITGB4/JUP, high-ITGA3/CD9 and high-CDK2/CDKN1A). A false-negative case had a tumor size of $38 \mathrm{~mm}$, suggesting the higher diagnostic reliability of the triple-positive status (high-ITGB4/JUP, high-ITGA3/CD9 and high- $C D K 2 / C D K N 1 A$ ) in the early detection of distant metastasis.

$D S D$. The Cox proportional hazards model found double-positive (high-ITGA3/CD9 and high-CDK2/CDKN1A) and positive margin to be independent significant variables (Table IIID). The cumulative survival was represented by the K-M curves between the groups of double-positive cases (high-ITGA3/CD9 and high-CDK2/CDKN1A) and the remaining negative cases according to size $(\leq 20$ or $>20 \mathrm{~mm})$ (Fig. 1D). The risk of DSD was significantly higher in double-positive cases (high-ITGA3/CD9 and high-CDK2/CDKN1A) with a small influence from tumor size. Positive margin was associated with fatality in double-positive cases (high-ITGA3/CD9 and high-CDK2/CDKN1A) (data not shown).

\section{Discussion}

In our previous study, we demonstrated that there are two OSCC metastatic traits: Simple LNM and uncontrollable locoregional spread and distant metastasis via the circulation, leading to fatality. We proposed that a biomarker system using integrin gene expression ratios of high-ITGA3/CD9 for locoregional spread and high-ITGB4/JUP for hematogenous dissemination is effective for distinguishing these two types of risks (3). With the aim of improving diagnostic reliability, RT-qPCR was performed to determine the expression levels of 11 genes of cyclin-related proteins (cyclins, CDKs and CDKIs) for calculating the gene expression ratios of 66 gene combinations and assessed their diagnostic value in combination with the previously reported integrin gene expression biomarkers. Four gene expression ratios (ITGA3/CD9, ITGB4/JUP, CDK1/CDKN1B and $C D K 2 / C D K N 1 A$ ) are predictive of major clinical events with a high degree of reliability in OSCC patients.

Two gene expression ratio patterns, high-ITGA3/CD9 and high- $C D K 1 / C D K N 1 B$, reflected the LNM risk with a high degree of reliability. While LNM occurred in $~ 50 \%$ of OSCC cases, it may not respond to therapy in certain cases. Therefore, predicting the risk of LNM with biomarkers would be helpful in selecting optimal treatment. The finding that $<90 \%$ of patients with high-ITGA3/CD 9 and high- $C D K 1 / C D K N 1 B$ eventually had LNM irrespective of the primary tumor size (Fig. 1A) suggests that LNM can be detected before it becomes clinically evident.

As PSR is strongly influenced by clinical factors (such as site of the lesion and the extent of involvement within the oral cavity) and factors associated with treatment (such as positive margin), it is hard to conduct an accurate risk assessment prior to starting therapy. In the present study, the analysis using the Cox proportional hazard model showed that concurrent high-ITGA3/CD 9 and high-CDK2/CDKN1A and positive surgical margins are significant predictors of PSR (Table IIIB). This suggests that biological propensities are important underlying risk factors for PSR. By contrast, clear surgical margins reflect the relative success of surgery and therefore strongly affect PSR, but it cannot serve as a preoperative parameter for PSR risk assessment. As demonstrated by the K-M curve for PSR, the combination of high-ITGA3/CD 9 and high-CDK2/CDKN1A without consideration of another clinical factor estimates the risk of PSR well (Fig. 1B), and the ratios will therefore provide surgeons with useful information for determining the extent of surgical resection.

Distant metastasis was identified in $\sim 6.5 \%$ of patients (Table I), which is lower than the rate $(\sim 10 \%)$ observed in clinical settings. The low distant metastasis rate may be due to our definition of distant metastasis, in which distant metastasis following PSR was classified as PSR. Achieving a highly reliable estimate of the risk of distant metastasis based on tumor progression and histological findings is difficult and numerous patients may be receiving unnecessary postoperative chemotherapy due to this. The analysis using the Cox proportional hazard model showed that none of the clinicopathological parameters were significant risk factors for distant metastasis, while the combination of high-ITGB4/JUP, high-ITGA3/CD9 and high- $C D K 2 / C D K N 1 A$ was the only significant risk factor for distant metastasis. This suggests that there is a biological propensity for distant metastasis and it is difficult to predict distant metastasis based on clinical parameters and histological findings. Reliable analysis of distant metastasis risks will enable appropriate selection of chemotherapy, molecular-targeted therapy and/or immunotherapy for patients with highly malignant cancer in the early stage when maximum therapeutic effect is expected. In addition, it may eliminate unnecessary adjuvant chemoradiation in the majority of patients.

The combination of positve margins, high-ITGA3/CD9 and high-CDK2/CDKN1A were shown to contribute to the risk of DSD. The influence of tumor size on prognosis was relatively small. By contrast, as demonstrated by analyses using the Cox proportional hazard model and the K-M curve (Table IIID and Fig. 1D), a malignant propensity indicated by the status of high-ITGA3/CD9 and high-CDK2/CDKN1A plus successful surgical resection directly affected the prognosis.

The present study demonstrated that $C D K$ gene expression ratios, $C D K 1 / C D K N 1 B$ and $C D K 2 / C D K N 1 A$, may be used as parameters for assessing the risk of LNM, PSR, distant metastasis and DSD reliably, particularly in combination with integrin-related gene expression ratios, ITGA3/CD9 and ITGB4/JUP, whose value has been confirmed previously (3). The cell cycle is regulated by a mechanism involving the coordinated actions of CDK, cyclin and CDKI $(8,10)$. Dysregulated function of these molecules is the most frequently observed anomaly in malignant tumors $(7,8,11)$. CDK1 is the key factor in the $G_{2} / M$ phase transition and can be considered as a cell proliferation index. Upregulation of $C D K 1$ is observed in 
OSCC and its overexpression is associated with malignant behavior. CDK1 is therefore expected to be an indicator of poor prognosis $(6,12)$. CDK2 plays various roles in DNA synthesis, modulation of $\mathrm{G}_{2}$ progression and in $\mathrm{G}_{1} / \mathrm{S}$ transition (13). $C D K 2$ overexpression was shown to be associated with malignant biological behavior and a poor prognosis in patients with head and neck carcinoma (14). CDKN1B (p27) and CDKN1A (p21) are CDKIs belonging to the Cip/Kip family that inhibit kinase activity of the cyclin E/A-CDK2 complex, thereby blocking the $\mathrm{G}_{1} / \mathrm{S}$ transition and inducing apoptosis $(15,16)$. Downregulation and increased degradation of these CDKIs were observed in cancerous tissues and their association with malignant behavior of squamous cell carcinoma was also demonstrated (17-19). Specific associations of $C D K-C D K I$ gene expression ratios with major clinical events (LNM, PSR, distant metastasis and DSD) suggest that cell cycle regulation mechanisms involving these molecules have effects on invasion, dissemination and tumor growth.

The present study demonstrated the feasibility of using four combinations of CDK and integrin gene expression status as a simple and relatively inexpensive assessment of the risk of locoregional spread or hematogenous dissemination. Highly reliable risk assessment prior to treatment enables selection of optimal therapy and use of novel therapeutic strategies, thereby improving control rates. Further studies using a large group of subjects and prospective clinical studies, as well as improved understanding of biological mechanisms, are essential for showing the clinical value of the biomarker system and application in the clinical setting.

\section{Acknowledgements}

The present study was supported by the Japan Society for the Promotion of Science (grant no. 25463108). The manuscript was translated and edited by ThinkSCIENCE K.K., Tokyo, Japan.

\section{References}

1. International Agency for Research on Cancer: GLOBOCAN 2012: Estimated cancer incidence, mortality and prevalence worldwide in 2012. http://globocan.iarc.fr/Pages/fact_sheets_ population.aspx. Accessed December 30, 2014.

2. NCCN: Clinical practice guidelines in oncology head and neck cancers. Version 2. http://www.nccn.org/professionals/physician gls/f_guidelines.asp. Accessed December 30, 2014.

3. Nagata M,Noman AA, Suzuki K, Kurita H, Ohnishi M, Ohyama T, Kitamura N, Kobayashi T, Uematsu K, Takahashi K, et al: ITGA3 and ITGB4 expression biomarkers estimate the risks of locoregional and hematogenous dissemination of oral squamous cell carcinoma. BMC Cancer 13: 410, 2013.
4. Nagata M, Fujita H, Ida H, Hoshina H, Inoue T, Seki Y, Ohnishi M, Ohyama T, Shingaki S, Kaji M, et al: Identification of potential biomarkers of lymph node metastasis in oral squamous cell carcinoma by cDNA microarray analysis. Int J Cancer 106: 683-689, 2003

5. Kurokawa A, Nagata M, Kitamura N, Noman AA, Ohnishi M, Ohyama T, Kobayashi T, Shingaki S and Takagi R; Oral, Maxillofacial Pathology and Surgery Group: Diagnostic value of integrin alpha3, beta4 and beta5 gene expression levels for the clinical outcome of tongue squamous cell carcinoma. Cancer 112: 1272-1281, 2008

6. Chen X, Zhang FH, Chen QE, Wang YY, Wang YL, He JC and Zhou J: The clinical significance of CDK1 expression in oral squamous cell carcinoma. Med Oral Patol Oral Cir Bucal 20: e7-e12, 2015.

7. Bonelli P, Tuccillo FM, Borrelli A, Schiattarella A and Buonaguro FM: CDK/CCN and CDKI alterations for cancer prognosis and therapeutic predictivity. BioMed Res Int 2014: 361020, 2014

8. Malumbres $\mathrm{M}$ and Barbacid M: Cell cycle kinases in cancer. Curr Opin Genet Dev 17: 60-65, 2007.

9. Yamamoto E, Kohama G, Sunakawa H, Iwai M and Hiratsuka H: Mode of invasion, bleomycin sensitivity, and clinical course in squamous cell carcinoma of the oral cavity. Cancer 51: 2175-2180, 1983.

10. Malumbres M: Physiological relevance of cell cycle kinases. Physiol Rev 91: 973-1007, 2011.

11. Shintani S, Mihara M, Nakahara Y, Kiyota A, Ueyama Y, Matsumura T and Wong DT: Expression of cell cycle control proteins in normal epithelium, premalignant and malignant lesions of oral cavity. Oral Oncol 38: 235-243, 2002.

12. Chang JT, Wang HM, Chang KW, Chen WH, Wen MC, Hsu YM, Yung BY, Chen IH, Liao CT, Hsieh LL, et al: Identification of differentially expressed genes in oral squamous cell carcinoma (OSCC): Overexpression of NPM, CDK1 and NDRG1 and underexpression of CHES1. Int J Cancer 114: 942-949, 2005.

13. De Boer L, Oakes V, Beamish H, Giles N, Stevens F, Somodevilla-Torres M, Desouza C and Gabrielli B: Cyclin A/cdk2 coordinates centrosomal and nuclear mitotic events. Oncogene 27: 4261-4268, 2008.

14. Dong Y, Sui L, Tai Y, Sugimoto K and Tokuda M: The overexpression of cyclin-dependent kinase (CDK) 2 in laryngeal squamous cell carcinomas. Anticancer Res 21: 103-108, 2001.

15. Pérez-Sayáns M, Suárez-Peñaranda JM, Gayoso-Diz P, Barros-Angueira F, Gándara-Rey JM and García-García A: The role of p21Waf1/CIP1 as a Cip/Kip type cell-cycle regulator in oral squamous cell carcinoma (Review). Med Oral Patol Oral Cir Bucal 18: e219-e225, 2013.

16. Kudo Y, Kitajima S, Ogawa I, Miyauchi M and Takata T: Down-regulation of Cdk inhibitor p27 in oral squamous cell carcinoma. Oral Oncol 41: 105-116, 2005.

17. Bandoh N, Hayashi T, Takahara M, Kishibe K, Ogino T, Katayama A, Imada M, Nonaka S and Harabuchi Y: Loss of p21 expression is associated with p53 mutations and increased cell proliferation and p27 expression is associated with apoptosis in maxillary sinus squamous cell carcinoma. Acta Otolaryngol 125: 779-785, 2005.

18. Ohashi K, Nemoto T, Eishi Y, Matsuno A, Nakamura K and Hirokawa K: Expression of the cyclin dependent kinase inhibitor p21WAF1/CIP1 in oesophageal squamous cell carcinomas. Virchows Arch 430: 389-395, 1997.

19. Gao L, Gu W, Zheng J, Ren W, Chang S, Wang X, Li S, Song T, Huang $\mathrm{C}$ and Zhi K: Clinicopathological and prognostic significance of p27 expression in oral squamous cell carcinoma: A meta-analysis. Int J Biol Markers 28: e329-e335, 2013. 